\title{
Evaluating the value of persuasive technology and the role of teachers in a blended learning course for social work students
}

\author{
Monique M. J. Engelbertink $\mathbb{D}^{\mathrm{a}, \mathrm{b}}$, Saskia M. Kelders ${ }^{\mathrm{b}, \mathrm{c}}$, Kariene M. Woudt-Mittendorffa \\ and Gerben J. Westerhof ${ }^{b}$ \\ a Research Centre Educational Innovation \& Effectiveness, University of Applied Sciences Saxion, Deventer,

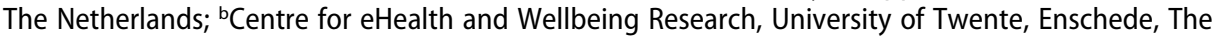 \\ Netherlands; 'Optentia Research Focus Area, North West University, Vanderbijlpark, South Africa
}

\begin{abstract}
Introduction: In this study, a blended learning course, designed using a participatory design approach and Persuasive Technology (PT), was evaluated by students and teachers. The use of a participatory design and PT may help facilitate a blended learning course that motivates students and supports teachers in this relatively new field. Method: Students received the blended course as part of their regular curriculum. Subsequently, interviews with students $(\mathrm{N}=10)$ and teachers $(\mathrm{N}=3)$, as well as a survey among students $(\mathrm{N}=35)$ were held to evaluate the blended course. Results: Most of the PT which was implemented motivated the students to participate in the online course. The techniques suggestions, similarity, reduction, self-monitoring and tunneling seemed to specifically motivate students. According to the students, their teachers sufficiently integrated the online course with the Face to Face Classes. Teachers stated that they stimulated intrinsic motivation, supported students to complete their tasks and facilitated the blended learning process (integrating the different elements of a blended learning course). Discussion/Conclusion: The use of PT motivated students. Teachers played a crucial role in merging different aspects of the blended learning course. Future challenges would be the implementation of social support online.
\end{abstract}

\section{ARTICLE HISTORY}

Received 29 March 2019

Accepted 8 January 2020

\section{KEYWORDS}

Persuasive technology; motivation; blended learning; social work; participatory design; reflection

\section{Introduction}

Blended learning, a current trend within higher education, can be defined as the integration of Face-to-Face-Classes (FTFC) combined with online activities used asynchronous (Garrison \& Kanuka, 2004; López-Pérez, Pérez-López, Rodríguez-Ariza, \& Argente-Linares, 2013). With the increasing possibilities of technology, blended learning has initiated didactic changes from teacher-driven to student-driven, increasing the possibility that students may become more active while learning online and also interacting more frequently with their teacher and fellow students online (de Jong, SavinBaden, Cunningham, \& Verstegen, 2014; Poon, 2013). Due to the blended learning method, students can take advantage from both the benefits of FTFC and online learning. 
Within FTFC, there is social interaction (Uğur, Akkoyunlu, \& Kurbanoğlu, 2011) and students can be guided by their teacher in forming their opinion (Todd et al., 2017). Whereas online learning offers a standardized learning environment regardless of the qualities of a particular teacher and the student can learn at any time, whereby the student can navigate back-and-forth to the learning content (Todd et al., 2017). Moreover blended learning offers a lot of potential in higher education, in that it allows students to learn at their own pace, in turn achieving better overall learning outcomes (Kiviniemi, 2014; Lowell Bishop \& Verleger, 2013), and delivering an effective and wellorganized learning environment (Garrison \& Kanuka, 2004; Kiviniemi, 2014; Schneider \& Preckel, 2017; Spanjers et al., 2015). The review of Bernard, Borokhovski, Schmid, Tamim, and Abrami (2014) confirms an increase in student achievement with blended learning compared to solely FTFC or solely distance learning methods. Additionally, the review by Todd et al. (2017), specifically focused on ethical courses, shows that blended learning method is preferable compared to the other methods due to the possibilities of adequately offering instructional and process-based content. Despite these promising results, it is a challenge to create a blended learning environment that engages and motivates students, as research has shown that one of the factors associated with students is that, at times, their self-regulation abilities may be insufficient (Bernard et al., 2014; Broadbent, 2017; Czerkawski \& Lyman, 2016; Schneider \& Preckel, 2017). Motivation is seen as a necessary, but not sufficient, precondition for engagement (Appleton, Christenson, \& Furlong, 2008). In the present study, the term 'motivation' was used stating that students have a 'desire or willingness to do something; [or demonstrate] enthusiasm' (English Oxford Online Dictionary). With the emergence of blended learning and online learning, the educational developer has been given the opportunity to use technology in order to keep students engaged and motivated throughout the learning process (Maple, Jarrott, \& Kuyini, 2013). However, it is important to ask: How can technology be used to motivate students to work in an online course of blended learning? In this study, we attempt to answer this through evaluating a blended learning course for social work students who made use of Persuasive Technology (PT) (see next paragraph). This research attempts to fill a gap in research associated with blended learning within the social work domain (Ayala, 2009; Maple et al., 2013).

Teachers and designers want the students to follow the course as intended, providing them with the best learning opportunities possible. Technology itself is able to contribute to this (Kelders, Kok, Ossebaard, \& Van Gemert-Pijnen, 2012). PT is the use of technology in eLearning or blended learning to positively influence the behaviors and attitudes of students, without using coercion, to increase the chances that students would follow an online course or intervention in the desired way (Oinas- Kukkonen \& Harjumaa, 2009; Van GemertPijnen, Kelders, Beerlage- De Jong, \& Oinas- Kukkonen, 2018). The aim of PT is thus to stimulate the interactivity and involvement of users (Oinas- Kukkonen \& Harjumaa, 2009). Examples of PT are self-monitoring where the (computer) system shows the user the status of his current actions or knowledge and praise where the (computer) system gives the user feedback about his tasks via symbols, pictures or words (Oinas- Kukkonen \& Harjumaa, 2009). The Persuasive Systems Design-model (PSD- model) is a model designed by OinasKukkonen and Harjumaa (2009) to categorize various persuasive techniques. According to the PSD-model, there are four categories of PT that can support users, in this case students, following a blended course. The categories included: Primary Task Support; Dialogue 
Table 1. Persuasive systems design-model (Oinas- Kukkonen \& Harjumaa, 2009).

\begin{tabular}{|c|c|c|c|}
\hline \multicolumn{4}{|c|}{ Persuasive Systems Design- model } \\
\hline Primary Task Support & Dialogue Support & Credibility Support & Social Support \\
\hline $\begin{array}{l}\text { Reduction, Tunneling, } \\
\text { Tailoring, } \\
\text { Personalization, Self- } \\
\text { monitoring, Simulation, } \\
\text { Rehearsal. }\end{array}$ & $\begin{array}{l}\text { Praise, Rewards, } \\
\text { Reminders, } \\
\text { Suggestion, } \\
\text { Similarity, Liking, } \\
\text { Social role }\end{array}$ & $\begin{array}{c}\text { Trustworthiness, Expertise, } \\
\text { Surface credibility, Real- } \\
\text { world feel, Authority, } \\
\text { Third- party, Verifiability }\end{array}$ & $\begin{array}{l}\text { Social learning, Social comparison, } \\
\text { Normative influence, Social } \\
\text { facilitation, Cooperation, } \\
\text { Competition, Recognition }\end{array}$ \\
\hline
\end{tabular}

Support; System Credibility Support; and Social Support (Table 1). Each category comprised a number of techniques that could be implemented in various ways in the online course, thus meeting the needs for support within the target group.

Although its use is relatively new, PT has been successfully applied within eHealth applications (Van Gemert-Pijnen et al., 2018). However, within the education field PT is used less, though its potential has been recognized (Burri Gram-Hansen \& Ryberg, 2013; Mintz \& Aagaard, 2012). Moreover, although PT is only mentioned sparingly in this context, techniques that are classified as PT have already been used in education. Maple et al. (2013), for example, used various Primary Task Support techniques associated with the PSD-model in order to engage their students, without using these terms. They trained their students in a structured way through online tasks (tunneling), appointed important milestones in participation of the online course to reduce frustration or delay as much as possible (reduction) and further, encouraged the students to develop their own learning style (tailoring). Finally, adding quizzes to the online part of blended learning can be referred to as the self-monitoring PT because the answers to the quizzes given by the computer give students feedback on their current level of knowledge. Adding quizzes, tests and self-assessments positively influences the effectiveness and attractiveness of blended learning (Spanjers et al., 2015).

In addition to the focus on the role of technology, the responsibility of the teacher in engaging and motivating students in a blended learning course is recognized as vital (Engelbertink, Kelders, Woudt- Mittendorff, \& Westerhof, under review; Maarop \& Embi, 2016). New competences were expected from teachers which were associated with different aspects of the blended learning course (Campbell, Davis, Phelan, \& Hanley, 2018; Czerkawski \& Lyman, 2016; Maarop \& Embi, 2016). The review of Maarop and Embi (2016) indicated that teachers found it difficult to maintain the right balance between the time they spent on the online course and the FTFC, that they lacked pedagogical and technical skills, and experienced more stress due to the increased workload. In order to reflect on the teachers' attitudes and working methods, it was vital that teachers were trained during the implementation of blended learning courses. The need to be familiar with technology as an instructor and to have enough time to solve technological problems were confirmed as vital in a recent study in the field of social work by Hafford-Letchfield, Dayananda, and Collins (2018).

\section{Aim of the study}

The course was developed using a participatory design. In a participatory design, designers, stakeholders and future users work together in a design process with the aim 
of, among other things, increasing support among stakeholders, increasing usability and engagement of users (Bratteteig \& Wagner, 2016; Simonsen \& Hertzum, 2012; Steen, 2013), which has also been an advocated approach for designing educational materials (Könings, Seidel, \& van Merriënboer, 2014). For the details of the current design, see Engelbertink et al. (under review). With the next phase being to analyze the course in real life among students and teachers to assess whether it came across as previously anticipated. The aim of the current study is to evaluate the added value brought by PT and the role in which the teacher played in the blended learning course. The research questions are: RQ1. To what extent in the online course did students evaluate persuasive techniques as motivating? And according to the students, which persuasive techniques could be further added to the existing online course to increase their motivation? and RQ2. How did the students and teachers evaluate the blending of the online and face-to-face components of the course and which improvements do they recommend?

\section{Method}

\section{Design}

Interviews and an online survey were conducted with the students. Both methods focused on the students' experiences with the course which incorporated PT, their preferences on additional PT related to a redesign of the course (RQ1) and the way in which blended learning was established (RQ2). Additionally, interviews with teachers were held that focused on the way that elements of the course were blended in practice (RQ2).

\section{Setting}

The blended learning course in this study was developed in a previous study (Engelbertink et al., under review) using a participatory design with 17 stakeholders (students, teachers, professionals, policymakers, instructional designers) at the School of Social Work at a University of Applied Sciences (UAS) in the East of the Netherlands. During six meetings with all stakeholders, the blended learning course was developed and improved in an iterative process. The stakeholders were able to provide feedback and propose new features from their own experience with online modules. The assumption was that a participatory design could lead to a blended learning course that is motivating according to all stakeholders and the used PT could stimulate the students while using the online part of the blended learning course (Engelbertink et al., under review). During the design process the stakeholders decided which techniques were used to design the blended learning course. During the development of the online part of the course it became clear which PT could not be implemented due to technical limitations of the software used. In sum, a lot of the techniques from the first three categories of the PSDmodel were used (Tables 3-5). The category Social Support did not fit with the content of the course (autobiographical reflection) because according to the majority of stakeholders, these techniques could disrupt the autobiographical writing process because of the competitive and group dynamic processes that are not desirable at the start of this individual reflection task (Engelbertink et al., under review). In the current evaluation of the course the students were asked to what extent the used PT motivated them and 
whether they would find some of the not used PT desirable in a redesign (see the section Materials and Tables 3-6). With the results of this evaluation, the next phase of the design process is started, namely a redesign of the blended learning course. The redesign of the course falls outside the scope of this article.

The aim of the blended course, evaluated in the current study, was to strengthen the professional identity of students by writing, and reflecting on their autobiography. The blended course is structured as follows. The students followed seven FTFC and after each class they met with a peer group of three other students. In these peer groups, the students interviewed each other asking questions based on their autobiographical writing within the online course. The peer group meetings were not pre-scheduled. After the peer group meeting, the students were expected to complete their online homework. The homework consisted of six themes relating to the autobiographical reflection method which was provided in the online course. The course was piloted in this study for 3rd and 4 th year students whose minor focused on exploring philosophical questions on a personal and professional level. The blended learning course was taught by experienced teachers $(\mathrm{N}=3)$, each with 16 students in their class $(\mathrm{N}=48)$. These teachers have experience in teaching autobiographical reflection and integrating FTFC, peer groups and homework assignments, but they have no experience in integrating FTFC with an online course (blended learning). The teachers received a teacher's manual and a protocol on how they could optimally blend the different elements of the blended course (FTFC, peer groups and the online course). The students were rewarded with course credits made up of 84 study hours. For students to achieve these credits, their teacher had to receive the students' autobiography on paper or by e-mail, two weeks after completing the FTFC. The teacher then marked the autobiography as 'accomplished' or 'not accomplished'.

\section{Interviews}

\section{Participants}

Ten undergraduate students who enrolled on the blended course (eight women and two men) participated in the interviews. In general, they had received little to no blended learning courses within their curriculum. The students had not previously followed an online course with PT either. This was also the first time that they had participated in a course based on autobiographical reflection. The students were from four different branches of studies (seven from social work, one from nursing, one from creative arts and technology, and one from psychology). Their mean age was 22.9 (min. age 21-max. 25).

The three teachers who taught the blended course (one woman and two men) also participated in the interviews. As mentioned before, these teachers had no prior experience with blended learning, however they were used to having students complete homework in preparation for a lesson. The male respondent was 51 years old and had a bachelor's degree. The other two respondents were both 42 years old and both had a master's degree.

\section{Materials}

The student participants were asked to match 23 cards each with one persuasive technique from three options: motivating, neutral, or not motivating. The cards 
represented 22 of the 28 PT techniques associated with the PSD-model. Appendix 1 provides an overview of the PT and the statements that were used during the interviews. Some cards combined two similar techniques, whereas other techniques were formulated by different statements on two separate cards. The statements related to the first four categories answered RQ1, with a fifth category, blended learning, being facilitated to answer RQ2. When a student marked a statement as 'not motivating', they were asked what it was about the technique that made them feel this way. Not all of the techniques were applied within the blended course. Regarding these techniques, students were asked if they thought that it would be possible for the techniques to be considered as motivational if they were to be applied in a redesign of the course.

The interviews with the three teachers were semi-structured and focused on the following two topics: content of the online course and blended learning. The results produced in the interviews were used for answering RQ2. Some exemplar questions were: what do you think of the online course? (content); to what extent did you discuss the online assignments in your lessons? (blended learning); to what extent do you want to communicate with students via the online course? (blended learning); what are possible improvements for the online course? (content).

\section{Procedure}

The interviews were conducted with the students, who were in pairs, by three fourth year bachelor students studying social work, and the first author. The first author was the initial interviewer of the first six interviews with students which assisted in supporting the students and enhanced their interview skills. The other four interviews with the respondents were conducted independently by the fourth year students. The fourth year students then interviewed the teachers in pairs, without the presence of the first author which helped stimulate the openness of the teachers, as the first author was a colleague of theirs. The interviews lasted approximately one hour and were all audio recorded.

\section{Data analysis}

The interviews were all verbatim transcribed. The coding scheme contained themes and subthemes constructed deductively by the statements and their prescribed answers (motivating, neutral, not motivating). The coding scheme contained also subthemes constructed inductively by analyzing the students' explanations behind their answers (Hofste, Boshoven, \& Munsterhuis, 2016).

\section{Survey}

\section{Participants}

Bachelor students $(\mathrm{N}=48)$ who followed the blended course, were invited to complete the survey. Of the 48 students approached, 35 (73\%) completed the survey (only the surveys that were fully completed have been included). Five participants were male (14\%), with the remaining 30 being female (86\%). This corresponded to the male/ female ratio throughout the entire program. The mean age was 23.2 years $(\mathrm{SD}=1.9)$. 30 students $(83 \%)$ followed a social work study, and six (17\%) participants followed another study program at this UAS, among which nursing, psychology and creative arts and technology 


\section{Materials}

The questionnaire included: one question about the technical operation of the online course; 19 statements about the evaluation of the PT used in the online course; 13 statements about any desired improvements of the PT in the online course; seven statements about the role of the teacher, including the way different elements of the blended course were integrated; and their opinion about the added value of the peer groups. Appendix 1 provides an overview of the PT and the statements that were used throughout the survey. Regarding the design of the interviews, the statements associated with the first four categories were used for answering RQ1, and statements in the fifth category, blended learning, answered RQ2. According to the development group, the PT from the third category, System Credibility Support, was required as a precondition for the online course. These were techniques that did not motivate the students as highly, though were necessary as a precondition for enabling their ability to follow the content properly (Engelbertink et al., under review). For this reason, within the third category participants were able to answer each statement with 'agree' versus 'disagree', rather than using the terms 'motivating' versus 'not motivating'. In an iterative process, the statements that were used during the survey were improved on the basis of the results produced in the interviews. Often, more statements on a particular PT strategy were used to gain a more complete understanding of the student's preferences during the survey. For example, for the technique 'reminders' different statements were formulated which were associated with alerting the student controlled by the used learning management system Blackboard, the teacher or through a WhatsApp group.

\section{Procedure}

Qualtrics, a tool to design an online survey, was used. The first author visited a lecture which covered all three classes during which the students filled out the survey. Any students who were absent were invited to complete the survey by e-mail.

\section{Data analysis}

SPSS was used to descriptively analyze the data.

\section{Results}

Before discussing the results of the two research questions, we first review the general experiences that students have mentioned.

\section{General experiences}

Table 2 presents the results of the students' general experiences upon completion of the survey. Overall, the students expressed that they were satisfied with the blended learning course and that they found the procedure used within the online element of the course clear. The alternation between theory, tips, examples, images and questions was sufficient. These results were similar to the opinions expressed by the students who were interviewed too. As a student remarked: 'I thought it was a nice course in the sense that there was a good variation between questions and information, not that you were 
Table 2. General experiences survey $(\mathrm{N}=35)$.

\begin{tabular}{lcccccc}
\hline Statements & TA $^{\text {a }}$ & A & N & D & TD & NO \\
\hline Clear operation online course & 23 & 48 & 20 & 8 & 0 & 0 \\
Alternation between theory, tips, examples, images and questions was sufficient & 11 & 69 & 20 & 0 & 0 & 0 \\
\hline
\end{tabular}

Legend: $\mathrm{TA}=$ totally agree, $\mathrm{A}=$ agree, $\mathrm{N}=$ neutral, $\mathrm{D}=$ disagree, $\mathrm{TD}=$ totally disagree, $\mathrm{NO}=$ no opinion. a results in \%.

Table 3. Results category primary task support.

\begin{tabular}{|c|c|c|c|c|c|c|c|c|c|c|}
\hline & & \multicolumn{9}{|c|}{ Evaluation (results in \%) } \\
\hline & & \multicolumn{4}{|c|}{ Interviews $(\mathrm{N}=10)$} & \multicolumn{5}{|c|}{ Survey $(\mathrm{N}=35)$} \\
\hline \multicolumn{2}{|c|}{ Primary Task Support } & M & $\mathrm{N}$ & NM & DM & M & $\mathrm{N}$ & NM & DM & NO \\
\hline Reduction & Divide assignments & 80 & 20 & 0 & 0 & 63 & 34 & 0 & 0 & 3 \\
\hline \multirow[t]{2}{*}{ Tunneling } & Guided step by step & 70 & 20 & 10 & 0 & 54 & 34 & 9 & 3 & 0 \\
\hline & Cannot go to next theme & 40 & 20 & 40 & 0 & 14 & 20 & 43 & 14 & 9 \\
\hline \multirow[t]{2}{*}{ Tailoring } & Choose assignments $^{a}$ & 80 & 20 & 0 & 0 & 63 & 14 & 11 & 3 & 9 \\
\hline & Choose methods ${ }^{a}$ & - & - & - & - & 69 & 17 & 3 & 0 & 11 \\
\hline \multirow[t]{2}{*}{ Persona-lisation } & Computer speaks to me & 50 & 40 & 10 & 0 & 46 & 46 & 6 & 0 & 3 \\
\hline & Design the course $e^{b}$ & 50 & 40 & 10 & 0 & 34 & 37 & 14 & 6 & 9 \\
\hline Self- monito-ring & See my own progress & 80 & 20 & 0 & 0 & 62 & 29 & 0 & 6 & 3 \\
\hline Simula-tion & What offer assignments ${ }^{a}$ & 50 & 30 & 10 & 0 & 54 & 31 & 9 & 0 & 6 \\
\hline Rehearsal & Methods are repeated & 40 & 50 & 10 & 0 & 29 & 31 & 17 & 17 & 6 \\
\hline
\end{tabular}

Legend: $\mathrm{M}=$ Motivating, $\mathrm{N}=$ Neutral, $\mathrm{NM}=$ Not motivating, $\mathrm{DM}=$ Demotivating, $\mathrm{NO}=$ No opinion/ not asked during the interview.

${ }^{\text {a }}$ The italicized statements relate to the techniques that were not applied in the online module. The students were asked whether they would find these desirable in a redesign.

${ }^{\mathrm{b}}$ This technique is not applied due to limited possibilities of the software.

Table 4. Results category dialogue support.

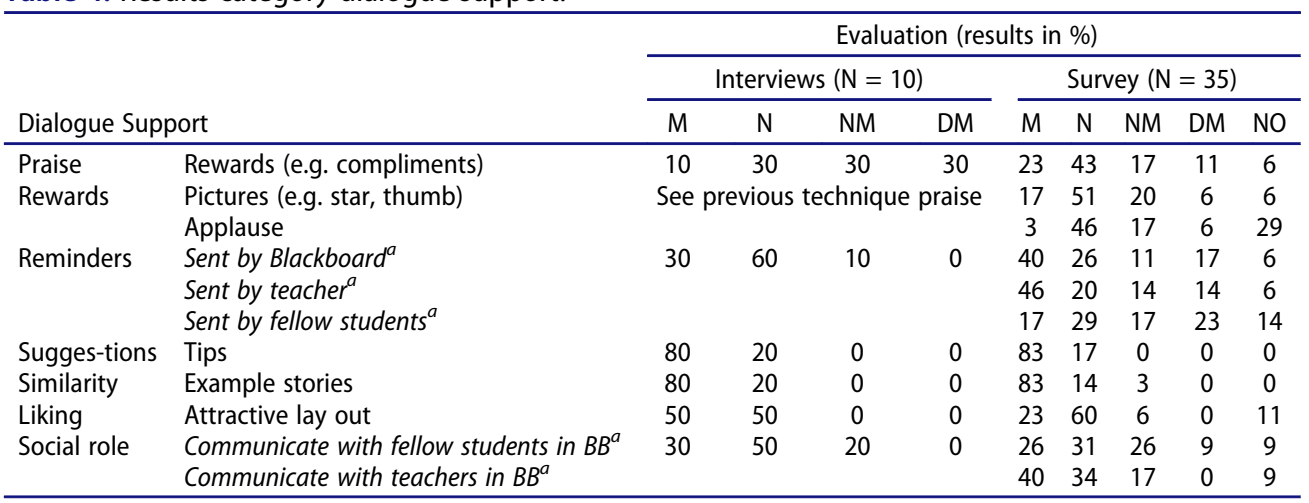

Legend: $\mathrm{M}=$ Motivating, $\mathrm{N}=$ Neutral, NM = Not motivating, $\mathrm{DM}=$ Demotivating, $\mathrm{NO}=$ No opinion.

${ }^{a}$ The italicized statements related to the techniques that were not applied in the online module. The students were asked whether they would find these desirable in a redesign.

${ }^{b}$ In the redesign this technique is not applied due to limited possibilities of the software.

answering questions all the time. But you also got some input in between about how you could think about it. I found that very pleasant.'

A question asked in the survey which queried whether the blended course contributed to the development of the students' (professional) identity demonstrated that $66 \%$ of the students answered 'yes', 17\% answered 'no' and 17\% answered 'I do not know'. When students indicated that the components of blended learning course had contributed, they were then asked to state the extent that this was the case. On average, they indicated that 
Table 5. Results category system credibility support.

\begin{tabular}{|c|c|c|c|c|c|c|c|c|c|c|c|}
\hline & & \multicolumn{10}{|c|}{ Evaluation (results in \%) } \\
\hline & & \multicolumn{4}{|c|}{ Interviews $(\mathrm{N}=10)$} & \multicolumn{6}{|c|}{ Survey $(\mathrm{N}=35)$} \\
\hline \multicolumn{2}{|c|}{ System Credibility Support } & M & $\mathrm{N}$ & NM & DM & $\mathrm{T}$ & A & $\mathrm{N}$ & D & TD & NO \\
\hline Trust- worthiness & Reliability and credibility & 60 & 40 & 0 & 0 & 17 & 37 & 29 & 14 & 0 & 3 \\
\hline Expertise & Expertise and professionalism & 60 & 30 & 10 & 0 & 11 & 34 & 34 & 20 & 0 & 3 \\
\hline Verifia-bility & Verify sources & 20 & 60 & 20 & 0 & 0 & 34 & 37 & 0 & 0 & 29 \\
\hline
\end{tabular}

Legend: $\mathrm{M}=$ Motivating, $\mathrm{N}=$ Neutral, $\mathrm{NM}=$ Not motivating, $\mathrm{DM}=$ Demotivating, $\mathrm{TA}=$ totally disagree, $\mathrm{A}=$ agree, $\mathrm{N}=$ neutral, $\mathrm{D}=$ disagree, $\mathrm{TD}=$ totally disagree, $\mathrm{NO}=$ No opinion.

Table 6. Results category social support.

\begin{tabular}{|c|c|c|c|c|c|c|c|c|c|c|}
\hline \multirow[b]{3}{*}{ Social Support } & & \multicolumn{9}{|c|}{ Evaluation (results in \%) } \\
\hline & & \multicolumn{4}{|c|}{ Interviews $(\mathrm{N}=10)$} & \multicolumn{5}{|c|}{ Survey $(\mathrm{N}=35)$} \\
\hline & & M & $\mathrm{N}$ & NM & DM & M & $\mathrm{N}$ & NM & DM & NO \\
\hline Social learning & Learning of others learning process ${ }^{a}$ & 40 & 30 & 30 & 0 & 29 & 34 & 31 & 3 & 3 \\
\hline Social comparison & View progress of others ${ }^{b}$ & 10 & 30 & 50 & 0 & 23 & 23 & 31 & 17 & 6 \\
\hline \multirow[t]{2}{*}{ Social facilitation } & Online feedback from students ${ }^{a}$ & 40 & 40 & 20 & 0 & 29 & 26 & 31 & 11 & 3 \\
\hline & Online feedback from teachers ${ }^{a}$ & & & & & 46 & 29 & 20 & 3 & 3 \\
\hline Cooperation & Collaborate with fellow students ${ }^{a}$ & 30 & 30 & 40 & 0 & 31 & 26 & 34 & 6 & 3 \\
\hline
\end{tabular}

Legend: $\mathrm{M}=$ Motivating, $\mathrm{N}=$ Neutral, NM = Not motivating, $\mathrm{DM}=$ Demotivating, $\mathrm{NO}=$ No opinion.

${ }^{a}$ The italicized statements related to the techniques that were not applied in the online module. The students were asked whether they would find these desirable in a redesign.

FTFC contributed 37\% overall, writing their autobiography as 34\%, conversations with family, loved ones and friends as $19 \%$, and their peer groups contributed only $7 \%$.

\section{RQ1 evaluation PT techniques}

Table 3 presents the results of the category Primary Task Support. According to the survey, more than half of the students found the following techniques motivating: reduction, tunneling (guided step-by-step) and self-monitoring. These results were similar to the opinions stated in the student interviews. A majority of the students did not feel motivated by the requirement to complete a theme before being able to start the next theme (second application of tunneling). A particularly apathetic technique was rehearsal, the students argued. The PT that were able to motivate more than half of the students in an improved version of the course were tailoring and simulation. The students stated in the interviews that tailoring made the course more personal and that they found it motivating when they were able to choose a reflection method that fitted well with their autobiography. The students further described it as motivating when they were reminded of what the assignments were able to offer them in terms of professional identity development (simulation).

Table 4 presents the results of the Dialogue Support category. According to the survey, the techniques in which over half of the students found motivating were suggestions (tips for making the assignments) and similarity (reading example stories from other students). These results were, again, similar to the opinions elicited during the students' interviews. Throughout the interviews, students expressed that these techniques gave them a sense of clarity as well as guidance. Due to technical limitations of the ICT tool Articulate Storyline, which was used to design the online course, it was not possible to 
implement the techniques reminders and social role. Therefore the students were asked how motivating they would find both techniques in a new course design: reminders sent by Blackboard or teachers or fellow students and communicate with fellow students in Blackboard and communicate with teachers in Blackboard (social role). The results of the survey show that students prefer both techniques related to their teachers than to their fellow students. Further, the survey found that most of the students described the PT rewards, pictures (praise) and applause (praise) as neutral. The students found rewards, like written compliments including 'well done', less motivating in comparison to praise techniques (pictures with thumbs up or a star/medal/hearing applause). However, three of the six students stated in their interview that they did not find praise or rewards particularly motivating. One student described the techniques as 'childish' and another called them 'disgusting'. They expressed that they preferred to receive the compliments personally.

According to most of the students during the survey and interviews, the online course sustained the category System Credibility Support (Table 5). Most of the students found the course trustworthy and stated that it exuded expertise and professionalism.

Table 6 presents the students' opinions corresponding with the possible application of the techniques within the Social Support category. Equal results were produced across the three answers (motivating, neutral, not motivating). Therefore, we can say that the opinions of the students were all dissimilar to each other, and also in comparison to the other categories. The technique that the students preferred most, according to the interviews and survey, was social facilitation (for the application: get online feedback on a specific development of the teacher, $46 \%$ ). Generally, the following techniques do not motivate or even demotivate the students who participated in the survey: social learning (34\%); social comparison (48\%); social facilitation (for the application: receive online feedback from students, 44\%); cooperation (40\%). Among the five students who found social learning and comparison not motivating, expressed in the interview process, they emphasized that writing your autobiography is a personal process and that they needed sufficient time and energy to write their own story. The exchanges throughout the FTFC were specifically used to gain an insight into one another's learning processes. Students also explained that they did not always want other students to read their life stories, due to confidentiality. Furthermore, students were anxious of competition and pressure when they were able to evaluate how far others were with their homework (social comparison). As we have seen in the techniques reminders and social role, they in turn motivated the students most when the technique social facilitation was performed by the teacher instead of by a fellow student. The interviews produced results stating that most students were motivated by social learning, if this will be applied in a redesign (Table 6). As a student remarked: 'If you see what added value others have experienced, then you also start thinking for yourself what this means for you.'

\section{RQ2 evaluation role of the teacher}

Table 7 presents the results of blended learning and the role of the teacher. The survey indicated that the online course was well integrated in relation to the FTFC by the teachers. Most of the students who took part in the survey agreed that the teacher sufficiently addressed the amount of effort that the students made in the online course, 
Table 7. Results blended learning support.

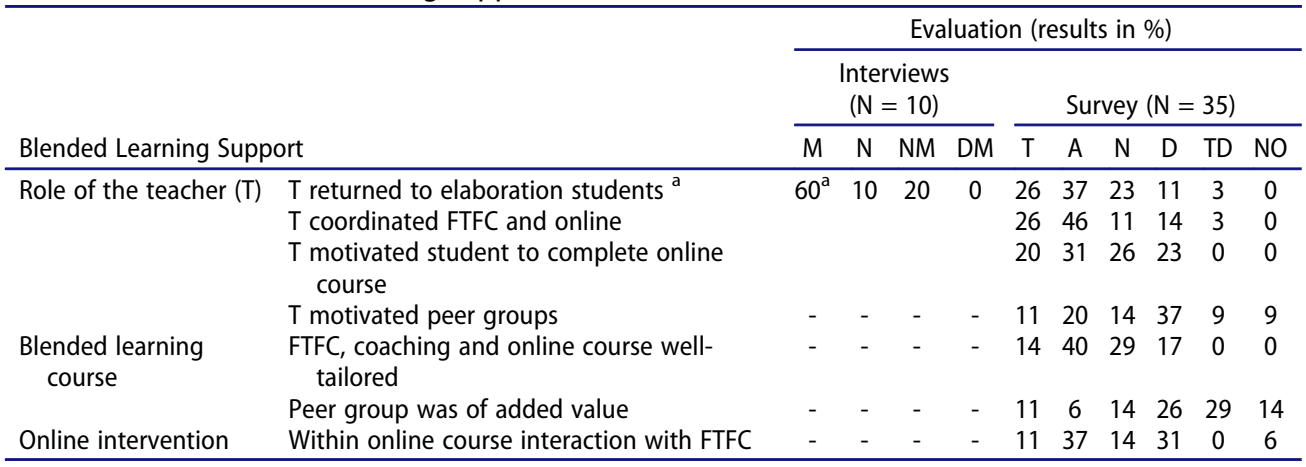

Legend: $M=$ Motivating, $N=$ Neutral, $N M=$ Not motivating, $N O=$ No opinion, $T A=$ totally disagree, $A=$ agree, $\mathrm{N}=$ neutral, $\mathrm{D}=$ disagree, $\mathrm{TD}=$ totally disagree,$-=$ not asked during the interview.

a During the interview this statement has been submitted: the teacher returns to the assignments made.

and the teacher ensured there was appropriate coordination between the classroom lessons and the online course. During the interviews, five of the ten students mentioned that their teacher encouraged them to work on the online course by mentioning it throughout the FTFC. One stated: 'You just know that during the lessons you will also be asked for it. (...) Yes actually, you are supported by that because it just comes back every lesson'. Some of them stated that they felt this lead to a more in-depth learning process. Four students indicated that their incentive to complete the online assignments came mainly from themselves. Three students also argued that the assignment was their own responsibility and that they did not require any stimulus from a lecturer. According to five students, teachers could explain the assignment in more detail and further, could return substantively to the assignment in the next lesson.

Subsequently, the teachers were asked what they did to motivate the students to complete their online assignments. Three didactic techniques were implemented: stimulating intrinsic motivation (asking: what did you learn?); supporting students on the task (asking them how far they are on the task at the beginning of the FTFC); and facilitating the blended learning (emphasize that keeping up with the online theme, parallel with the FTFC, offers an advantage in the learning process). A teacher indicated: ' I said in class, the assignments, did you finish them? How did that work for you? So the main thing is to emphasize the content. I hope that they become intrinsically motivated to actually do their homework.' Two of the three teachers followed the protocol each week to achieve the optimal blend between FTFC and the online course. The other lecturer, who did not follow the protocol exactly, indicated that he encouraged the students only ' $a$ limited amount' to follow the online course. He did not ask about their experiences with the homework at the start of each lesson, as the protocol had indicated to do so, as an attempt to encourage the students. However, throughout each lesson, he broached the content themes, explaining what the online course brought him personally. He encouraged them to only express something about their homework if they felt the need to do so, and to use their initiative when making this decision.

The results produced by the students (in interviews and in the survey) and by the teachers emphasized that the protocol had been sufficiently followed by the teachers 
regarding the integration between FTFC and the online course. However, the protocol did not seem to be followed by the teachers when encouraging the peer groups to come together. Almost half of the students did not feel that their teacher motivated them to come together in the peer group post-FTFC. During the survey, in relation to peer group activities, most of the students answered that they did not interview one another about their life stories. During the interviews, five out of ten students stated that they did not feel a need to come together in the peer group, as they had already discussed the topic of the lesson in an alternative environment (with friends and family or in FTFC). Some students indicated that they did not know each other well enough to feel comfortable with sharing their life story within their peer group: 'So I would have to tell my personal story in a coaching group in which I do not know anyone. And that just felt crazy for me'. The teachers seemed to agree with this explanation, however they characterized it also as the students demonstrating a lack of listening skills: 'So if you are in such a coaching group and there is not enough attention; you do not get together with each other to create the conditions for telling'. A more practical reason for not meeting with their peer group, according to one teacher and two students, was associated with time restrictions due to the tight schedule between lessons and tasks in other lessons.

\section{Conclusion and discussion}

First, we answered the first part of RQ1: To what extent in the online course did students evaluate PT as motivating? The course contained PT from three out of the four categories in the PSD-model: Primary Task Support, Dialogue Support and System Credibility Support based on a participatory design process (Engelbertink et al., under review). Overall, the PT techniques which were used were described by the students as motivating to a high degree. The techniques that were most motivating for the students were reduction, tunneling, self-monitoring (in Primary Task Support), and suggestions and similarity (in Dialogue Support). A focus on the primary task (Lehto \& Oinas-kukkonen, 2011) and dialogue support has often been found to occur within online eHealth interventions (Kelders et al., 2012) and the techniques of these categories are most frequently reported as motivated in eHealth (Asbjørnsen et al., 2019). Interestingly, a different application of the strategy tunneling was deemed as demotivating by a majority of students, indicating attention should not only be paid to which techniques to use, but also how they should be used. Furthermore, according to most of the students the online course sustained the category System Credibility Support.

The second part of RQ1: And according to the students, which PT could be further added to the existing online course to increase their motivation? can be answered as follows. The students indicated that the following PT was sufficient to motivate them in an improved version of the course: tailoring and simulation (in Primary Task Support), reminders and social role (communicate online with their teachers in Blackboard) (in Dialogue Support). The techniques within the fourth category, Social Support, did not strongly contribute to the students' motivation, thus it would not be beneficial to include them in a redesign of the course. Regarding the technique social comparison, the students were apprehensive about pressure and competition, which was in line with the motivation of the stakeholders not to include this technique (Engelbertink et al., under review). The potential risks of negative emotions produced by this technique were recognized by 
Suls, Martin, and Wheeler (2002). Besides this, students preferred to receive feedback from their teachers instead of feedback from fellow students (social facilitation) due to confidentially. This preference for receiving online feedback from a professional, rather than a peer, is also a strong finding in eHealth research among adults (Westerhof, Lamers, Postel, \& Bohlmeijer, 2017). Research in the educational field also showed that students (young adults and adults) prefer feedback from their teacher instead of their peers. Additionally, the group of adults do want feedback from their peers but then supplemented with feedback from the teacher (Motlagh, 2015). It could be that students feel worried that fellow students may not be able to give them appropriate feedback on their written autobiography. This may especially be a challenge if they only read a specific part of the autobiography, as students may be unable to read the life story of a fellow student from a holistic perspective. Though, in the light of research by Bluck and Levine (1998): Can fellow-students guide their peers to 'broaden and redefine the view of the self (p. 201)?

We now turn to answering RQ2: 'To what extent did the students and teachers find the course components optimally blended by the teachers and which improvements do they recommend?'. Overall, the students found the course to be blended adequately by the teachers, however improvements could be made by the teachers by ensuring they follow the protocol on a weekly basis: The teacher started the beginning of each FTFC by returning to the homework assignments which in turn motivated the students to do their homework and initiated the possibility of adding more depth to their assignments. Moreover, the end of each FTFC would prepare students for the next homework assignment. Additionally, teachers used different techniques suitable for supporting the students to do their homework, thereby enhancing academic engagement (Appleton et al., 2008). The teachers stimulated intrinsic motivation, as they supported students to complete their tasks, and stimulated the blended learning process (integrating the different elements of a blended learning course). The present study showed that teachers must be aware of the crucial role that they play in integrating the different elements of the course (merging the FTFC and the peer groups with the online course). The peer-groups seemed to be less integrated in the blended course. This may have been related to the reservations that students had on learning from peers in this particular context, yet it also demonstrates the important role played by the teacher. Teachers motivated students to a lesser extent to organize peer group meetings which may have diminished the perceived value of these groups for the students themselves. Schneider and Preckel (2017) indicated that small improvements could be made by teachers which would increase student performance. Among other things, they mentioned ensuring strong attendance in each class, clarity of the course objectives and indicating the student's responsibility in the course. Similar to Schneider and Preckel (2017), the skills that some of the teachers displayed in this study were instigated by minor improvements in instruction: encouraging students to do their homework by requesting it at the start of the lesson, and actively encouraging students to sit together in their peer groups. Research on eHealth has shown that the power of reminders is a highly contributory factor (Kelders et al., 2012) and sending an online reminder from an instructor in the education field can be seen as promoting engagement (Martin \& Bolliger, 2018). Since most students looked at this in a positive or neutral way, sending reminders online via a learning management system or by e-mail from the teacher could act as a motivating tool for stimulating the blended learning process. Additionally, our recommendation is to 
experiment with sending reminders within blended learning courses in social work education. However of course it is important to find a balance in student learning process between student independence and teacher support. So if sending reminders is desirable for students and these reminders can be implemented by a learning management system such as Blackboard or Canvas or via a mail system then that is worth trying.

In the introduction it was mentioned that motivation is an underlaying aspect of student engagement (Appleton et al., 2008). Student engagement is often seen as consisting of behavioral (the participation), cognitive (contemplation and willingness to make the effort), emotional (positive and negative reactions) and academic engagement (homework completion, time on task) (Appleton et al., 2008; Fredricks, Blumenfeld, \& Paris, 2004). Students provided explanations in the interview process regarding their level of motivation that could be linked to the various forms of engagement. The students additionally mentioned that they did not know each other well enough to come together in a peer group. This explanation suggests a lack of behavioral engagement. The PT suggestions and similarity gave students clarity and guidance which could be seen as supporting self-regulation; a part of cognitive engagement (Appleton et al., 2008). The PT social learning could also enhance cognitive engagement, as students mentioned that reading about the learning process of others made them think about what it means to be themselves. One third of the interviewed students expressed that praise and reward techniques evoked strong negative emotions, referring to a lack of emotional engagement. The PT social comparison did not motivate students to work on the online course (academic engagement), as they were afraid of competition, creating unnecessary pressure. The PT social facilitation could potentially increase academic engagement, since almost half of the students found it motivating to receive feedback from teachers. In conclusion, PT and blended learning seem to impact the different components of engagement. Future research could take advantage of this finding, and use PT and blended learning to specifically alter certain aspects of engagement. Moreover, asking open questions about motivation provided insight into various forms of engagement which could encourage teachers to initiate a conversation related to this topic.

\section{Limitations and future research}

An unexpected result produced during the course was related to a technical aspect of the ICT tool used. 'Articulate Storyline' was incapable of storing the students' autobiographical reflections in the web browser. Despite our extensive testing using different browsers, it did not work as it was expected to. Therefore, after a complaint by a student who lost her reflections and had to start again, the researchers immediately decided to advise the students to answer the questions from the online course in a separate text document. This was stated via an announcement on Blackboard. However, on day 3 of the course, the teachers had to explain this several times to students which was distracting for both teachers as well as the students.

In this study, we were able to investigate experiences with PT and evaluate the merging of different elements in the blended learning course. This research was limited to the opinion of students and teachers, as we did not measure learning outcomes or use learning analytics. The results encourage us to follow the path we have taken, in turn ensuring we implement the blended course into the second year social work students' curriculum. We 
developed a redesign of the blended learning course using the evaluation data to optimally motivate students. Further research using a Randomized Controlled Trial among our students will yield more insight into the engagement and motivation of the students using the course, its effectiveness and the role of PT in this respect. Further research should also be facilitated to evaluate whether students appreciate online communication (including feedback) from teachers more so than online communication with fellow students. Additionally, whether fellow students are able to provide meaningful feedback on their peers' autobiographic stories. Gaining feedback and (online) collaboration with teachers and peers can be motivating according to research and from some student's points of view (Bernard et al., 2014), though they may need some training (Maarop \& Embi, 2016). Follow up studies could investigate how to design social support in a blended learning course when the content of the course concerns an individual and privacy sensitive learning processes.

\section{Acknowledgments}

The authors would like to thank Deborah Hofste, Ilse Boshoven and Marije Munsterhuis for their interviews with the students and the teachers.

\section{Disclosure statement}

No potential conflict of interest was reported by the authors.

\section{Compliance with ethical standards}

Informed consent was obtained from all individual participants included in the study.

\section{Notes on contributors}

Monique M. J. Engelbertink is a $\mathrm{PhD}$ student of the University of Twente and a senior lecture of Saxion, University of Applied Sciences, the Netherlands. Her research interests are reflection, autobiographical writing and persuasive technology

Saskia M. Kelders is Assistant Professor at the Center for eHealth and Wellbeing Research at the University of Twente, the Netherlands and extraordinary professor at Optentia Research Focus Area, North-West University, South Africa. Her research interests are positive psychology, persuasive technology and digital interventions.

Kariene M. Woudt-Mittendorff is Associated Lector at Saxion, University of Applied Sciences, The Netherlands. Her research interests are teaching methods, career counseling and reflection.

Gerben J. Westerhof is Professor at Storylab of the Center for eHealth and Wellbeing Research of the University of Twente, the Netherlands. His research interests are narrative psychology and technology

\section{ORCID}

Monique M. J. Engelbertink (D) http://orcid.org/0000-0003-2246-436X 


\section{References}

Appleton, J. J., Christenson, S. L., \& Furlong, M. J. (2008). Student engagement with school: Critical conceptual and methodological issues of the construct. Psychology in the Schools, 45(5), 369-386.

Asbjørnsen, R. A., Smedsrød, M. L., Solberg Nes, L., Wentzel, J., Varsi, C., Hjelmesæth, J., \& van Gemert-pijnen, J. E. (2019). Persuasive system design principles and behavior change techniques to stimulate motivation and adherence in electronic health interventions to support weight loss maintenance: Scoping review. Journal of Medical Internet Research, 21(6), e14265.

Ayala, J. S. (2009). Blended learning as a new approach to social work education. Journal of Social Work Education, 45(2), 277-289.

Bernard, R. M., Borokhovski, E., Schmid, R. F., Tamim, R. M., \& Abrami, P. C. (2014). A meta-analysis of blended learning and technology use in higher education: From the general to the applied. Journal of Computing in Higher Education, 26(1), 87-122.

Bluck, S., \& Levine, L. J. (1998). Reminiscence as autobiographical memory: A catalyst for reminiscence theory development. Ageing and Society, 18(2), 185-208.

Bratteteig, T., \& Wagner, I. (2016). Unpacking the notion of participation in participatory design. Computer Supported Cooperative Work: CSCW: An International Journal, 25(6), 425-475.

Broadbent, J. (2017). Comparing online and blended learner's self-regulated learning strategies and academic performance. Internet and Higher Education, 33, 24-32.

Burri Gram-Hansen, S., \& Ryberg, T. (2013). Persuasion, learning and context adaptation. International Journal of Conceptual Structures and Smart Applications, 1(2), 28-37.

Campbell, J., Davis, M., Phelan, A., \& Hanley, D. (2018). Dealing with the learning needs of child welfare social and health care workers: An interdisciplinary approach to blended learning with part time students. Social Work Education, 37(6), 746-760.

Czerkawski, B. C., \& Lyman, E. W. (2016). An instructional design framework for fostering student engagement in online learning environments. TechTrends, 60(6), 532-539.

de Jong, N., Savin-Baden, M., Cunningham, A. M., \& Verstegen, D. M. L. (2014). Blended learning in health education: Three case studies. Perspectives on Medical Education, 3, 278-288.

Engelbertink, M. M. J., Kelders, S. M., Woudt- Mittendorff, K. M., \& Westerhof, G. J. (under review). Participatory design of persuasive technology in a blended learning course.

Fredricks, J. A., Blumenfeld, P. C., \& Paris, A. H. (2004). School engagement: Potential of the concept, state of the evidence. Review of Educational Research, 74(1), 59-109.

Garrison, D. R., \& Kanuka, H. (2004). Blended learning: Uncovering its transformative potential in higher education. Internet and Higher Education, 7(2), 95-105.

Hafford-Letchfield, T., Dayananda, A., \& Collins, D. (2018). Digital storytelling for interprofessional collaborative practice to develop quality and service improvements practice to develop quality and service improvements. Social Work Education, 37(6), 804-812.

Hofste, D., Boshoven, I., \& Munsterhuis, M. (2016). Blended learning in de opleiding social work. Enschede: Saxion, University of Applied Sciences.

Kelders, S. M., Kok, R. N., Ossebaard, H. C., \& Van Gemert-Pijnen, J. E. W. C. (2012). Persuasive system design does matter: A systematic review of adherence to web-based interventions. Journal of Medical Internet Research, 14(6), 1-24.

Kiviniemi, M. T. (2014). Effects of a blended learning approach on student outcomes in a graduate-level public health course. BMC Medical Education, 14, 47.

Könings, K. D., Seidel, T., \& van Merriënboer, J. J. G. (2014). Participatory design of learning environments: Integrating perspectives of students, teachers, and designers. Instructional Science, 42(1), 1-9.

Lehto, T., \& Oinas-kukkonen, H. (2011). Persuasive features in web-based alcohol and smoking interventions : A systematic review of the literature. Journal of Medical Internet Research, 13(3), $1-20$.

López-Pérez, M. V., Pérez-López, M. C., Rodríguez-Ariza, L., \& Argente-Linares, E. (2013). The influence of the use of technology on student outcomes in a blended learning context. Educational Technology Research and Development, 61(4), 625-638. 
Lowell Bishop, J., \& Verleger, M. (2013). The flipped classroom: A survey of the research. In Proceedings of the annual conference of the american society for engineering education (pp. 1-18). Atlanta. doi:10.1109/FIE.2013.6684807.

Maarop, A. H., \& Embi, M. A. (2016). Implementation of blended learning in higher learning institutions: A review of literature. International Education Studies, 9(3), 41.

Maple, M., Jarrott, H., \& Kuyini, A. B. (2013). Blended learning in rural social work education: Reflections from a new australian bachelor of social work course. Social Work Education, 32(3), 349-364.

Martin, F., \& Bolliger, D. U. (2018). Engagement matters: Student perceptions on the importance of engagement strategies in the online learning environment. Online Learning Journal, 22(1), 205-222.

Mintz, J., \& Aagaard, M. (2012). The application of persuasive technology to educational settings. Educational Technology Research and Development, 60(3), 483-499.

Motlagh, L. N. (2015). Who do learners prefer to be corrected by? Teachers or classmates? Procedia - Social and Behavioral Sciences, 199, 381-386.

Oinas- Kukkonen, H., \& Harjumaa, M. (2009). Persuasive systems design: key issues, process model, and system features. Communications of the Association for Information Systems, 24 (Article28), 485-500.

Poon, J. (2013). Blended learning: An institutional approach for enhancing students' learning experiences. Journal of Online Learning and Teaching, 9(2), 271-288. Retrieved from http://hdl. handle.net/10536/DRO/DU:30057995

Schneider, M., \& Preckel, F. (2017). Variables associated with achievement in higher education: A systematic review of meta-analyses. Psychological Bulletin, 143(6), 565-600.

Simonsen, J., \& Hertzum, M. (2012). Sustained participatory design: Extending the iterative approach. Design Issues, 28(3), 10-21.

Spanjers, I. A. E., Könings, K. D., Leppink, J., Verstegen, D. M. L., de Jong, N., Czabanowska, K., \& van Merriënboer, J. J. G. (2015). The promised land of blended learning: Quizzes as a moderator. Educational Research Review, 15, 59-74.

Steen, M. (2013). Virtues in participatory design: Cooperation, curiosity, creativity, empowerment and reflexivity. Science and Engineering Ethics, 19(3), 945-962.

Suls, J., Martin, R., \& Wheeler, L. (2002). Current directions in psychological science social comparison: Why, with whom, and with what effect? Current Directions in Psychological Science, 11(5), 159-163.

Todd, E. M., Watts, L. L., Mulhearn, T. J., Torrence, B. S., Turner, M. R., Connelly, S., \& Mumford, M. D. (2017). A meta-analytic comparison of face-to-face and online delivery in ethics instruction: The case for a hybrid approach. Science and Engineering Ethics, 23(6), 1719-1754.

Uğur, B., Akkoyunlu, B., \& Kurbanoğlu, S. (2011). Students' opinions on blended learning and its implementation in terms of their learning styles. Education and Information Technologies, 16(1), 5-23.

Van Gemert-Pijnen, J. E. W. C., Kelders, S. M., Beerlage - De Jong, N., \& Oinas - Kukkonen, H. (2018). Persuasive health technology. In J. E. W. C. Van Gemert-Pijnen, S. M. Kelders, H. Kip, \& R. Sanderman (Eds.), eHealth research, theory and development, a multidisciplinary approach (pp. 344). London: Routledge.

Westerhof, G. J., Lamers, S. M. A., Postel, M. G., \& Bohlmeijer, E. T. (2017). Online therapy for depressive symptoms: An evaluation of counselor-led and peer-supported life review therapy. The Gerontologist. doi:10.1093/geront/gnx140 